\title{
COFINITENESS AND FINITENESS OF GENERALIZED LOCAL COHOMOLOGY MODULES
}

\author{
LIZHONG CHU
}

(Received 9 October 2008)

\begin{abstract}
Let $I$ be an ideal of a commutative Noetherian local ring $R$, and $M$ and $N$ two finitely generated modules. Let $t$ be a positive integer. We mainly prove that (i) if $H_{I}^{i}(M, N)$ is Artinian for all $i<t$, then $H_{I}^{i}(M, N)$ is $I$-cofinite for all $i<t$ and $\operatorname{Hom}\left(R / I, H_{I}^{t}(M, N)\right)$ is finitely generated; (ii) if $d=\operatorname{pd}(M)<\infty$ and $\operatorname{dim} N=n<\infty$, then $H_{I}^{d+n}(M, N)$ is $I$-cofinite. We also prove that if $M$ is a nonzero cyclic $R$-module, then $H_{I}^{i}(N)$ is finitely generated for all $i<t$ if and only if $H_{I}^{i}(M, N)$ is finitely generated for all $i<t$.
\end{abstract}

2000 Mathematics subject classification: primary 13D45.

Keywords and phrases: local cohomology, generalized local cohomology, cofinite, Artinian.

\section{Introduction}

Let $R$ be a commutative Noetherian ring and $I$ a proper ideal of $R$. In 1969, Grothendieck proposed the following conjecture.

CONJECTURE 1.1. Let $N$ be a finitely generated $R$-module and let I be an ideal of $R$. Then $\operatorname{Hom}\left(R / I, H_{I}^{i}(N)\right)$ is finitely generated for all $i \geq 0$.

Hartshorne provided a counter-example to this conjecture in [9]. He defined an $R$-module $L$ to be $I$-cofinite if $\operatorname{Supp}_{R}\{L\} \subseteq V(I)$ and $\operatorname{Ext}_{R}^{i}(R / I, L)$ is a finitely generated $R$-module for any $i \geq 0$, where $V(I)$ denotes the set of prime ideals of $R$ containing $I$, and he asked the following question.

Question 1.2. Let $N$ be a finitely generated $R$-module and let $I$ be an ideal of $R$. Then is $H_{I}^{i}(N) I$-cofinite for all $i$ ?

In general, the answer is also no, even if $R$ is a regular local ring. See [6] for a counter-example to this question.

Supported by the National Natural Science Foundation (No. 10771152) of China, by the Research Foundation (No. Q4107805) of Suzhou University and by the Research Foundation of Pre-research Project (No. Q3107852) of Suzhou University.

(C) 2009 Australian Mathematical Publishing Association Inc. 0004-9727/2009 \$16.00 
The generalized local cohomology module

$$
H_{I}^{i}(M, N)=\underset{n}{\lim } \operatorname{Ext}_{R}^{i}\left(M / I^{n} M, N\right)
$$

for all $R$-modules $M$ and $N$ was introduced by Herzog in [10]. Clearly, it is a generalization of the usual local cohomology module. The study of generalized local cohomology modules was continued by many authors (see, for example, $[2,16]$ ). In [17], Yassemi asked whether Question 1.2 holds for generalized local cohomology. And the cofiniteness of generalized local cohomology modules is studied by DivaaniAazar and Sazeedeh [8] and Khashyarmanesh and Yassi [11].

At the same time, Conjecture 1.1 inspires us to think about the following question.

Question 1.3. Let $M$ and $N$ be two finitely generated $R$-modules. When is $\operatorname{Hom}\left(R / I, H_{I}^{i}(M, N)\right)$ finitely generated?

Asadollahi, Khashyarmanesh and Salarian [1] proved that if $H_{I}^{i}(M, N)$ is finitely generated for all $i<t$, then $\operatorname{Hom}\left(R / I, H_{I}^{t}(M, N)\right)$ is finitely generated. As an analogue of this result, we show that if $H_{I}^{i}(M, N)$ is Artinian for all $i<t$, then $H_{I}^{i}(M, N)$ is $I$-cofinite for all $i<t$ and $\operatorname{Hom}\left(R / I, H_{I}^{t}(M, N)\right)$ is finitely generated. We also prove that if $d=\operatorname{pd}(M)<\infty$ and $\operatorname{dim} N=n<\infty$, then $H_{I}^{d+n}(M, N)$ is $I$-cofinite, which is a generalization of [6, Theorem 3].

Throughout this paper, $(R, \mathfrak{m})$ is a commutative Noetherian local ring (with nonzero identity), $M$ and $N$ are finitely generated $R$-modules and $I$ is a proper ideal of $R$. We refer the reader to [3] or [4] for any unexplained terminology.

\section{Results}

We begin this section with some lemmas.

LEMMA 2.1. Let $M$ be a finitely generated $R$-module. If $H_{I}^{i}(M)$ is Artinian for all $i<t$, then $H_{I}^{i}(M)$ is $I$-cofinite for all $i<t$ and $\operatorname{Hom}\left(R / I, H_{I}^{t}(M)\right)$ is finitely generated.

Proof. This can be deduced from [7, Theorem 2.1] and [13, Proposition 4.3].

LEMMA 2.2. Let $M$ be a finitely generated $R$-module. If $L$ is Artinian and I-cofinite, then $\operatorname{Ext}_{R}^{i}(M, L)$ is I-cofinite for all $i$.

Proof. Since $L$ is Artinian, $\operatorname{Ext}_{R}^{i}(M, L)$ is Artinian for all $i$. By [13, Proposition 4.3], it suffices to prove that $\operatorname{Hom}_{R}\left(R / I, \operatorname{Ext}_{R}^{i}(M, L)\right)$ is finitely generated. In the following, we show that $\operatorname{Hom}_{R}\left(R / I, \operatorname{Ext}_{R}^{i}(M, L)\right)$ is of finite length. Since

$$
\begin{aligned}
\operatorname{Hom}_{R}\left(R / I, \operatorname{Ext}_{R}^{i}(M, L)\right) & \cong \operatorname{Hom}_{R}\left(R / I, \operatorname{Ext}_{R}^{i}(M, L)\right) \otimes \widehat{R} \\
& \cong \operatorname{Hom}_{\widehat{R}}\left(\widehat{R} / I \widehat{R}, \operatorname{Ext}_{\widehat{R}}^{i}(\widehat{M}, L)\right),
\end{aligned}
$$

we may assume that $R$ is $\mathfrak{m}$-adic complete. 
Set $E=E(R / \mathfrak{m})$, an injective envelope of $R / \mathfrak{m}$. By [15, Theorem 11.57],

$$
\operatorname{Hom}_{R}\left(\operatorname{Hom}_{R}\left(R / I, \operatorname{Ext}_{R}^{i}(M, L)\right), E\right) \cong R / I \otimes \operatorname{Tor}_{i}^{R}\left(M, \operatorname{Hom}_{R}(L, E)\right) .
$$

By Matlis duality, $R / I \otimes \operatorname{Tor}_{i}^{R}\left(M, \operatorname{Hom}_{R}(L, E)\right)$ is finitely generated, so it is enough to show that it is Artinian. Since $L$ is $I$-cofinite and Artinian, $\operatorname{Hom}_{R}(R / I, L)$ is of finite length, and then $\operatorname{Hom}_{R}\left(\operatorname{Hom}_{R}(R / I, L), E\right) \cong R / I \otimes \operatorname{Hom}_{R}(L, E)$ is of finite length. In particular,

$$
\operatorname{Supp}_{R}\left\{R / I \otimes \operatorname{Hom}_{R}(L, E)\right\}=V(I) \cap \operatorname{Supp}_{R}\left\{\operatorname{Hom}_{R}(L, E)\right\}=\{\mathfrak{m}\} .
$$

Therefore

$$
\operatorname{Supp}_{R}\left\{R / I \otimes \operatorname{Tor}_{i}^{R}\left(M, \operatorname{Hom}_{R}(L, E)\right)\right\} \subseteq V(I) \cap \operatorname{Supp}_{R}\left\{\operatorname{Hom}_{R}(L, E)\right\}=\{\mathfrak{m}\} .
$$

This completes the proof.

The following lemma plays a key role in the proof of our first main result.

LEMMA 2.3. Let $M$ be a finitely generated $R$-module ands be a nonnegative integer. Let $L$ be an $R$-module such that $H_{I}^{i}(L)$ is Artinian and I-cofinite for all $i<s$. If $H_{I}^{i}(M, L)$ is Artinian for all $i<s$, then $H_{I}^{i}(M, L)$ is I-cofinite for all $i<s$.

PRoof. The proof is by induction on $s$. When $s=1$, by the hypothesis, $H_{I}^{0}(L)$ is Artinian and $I$-cofinite. Then $\operatorname{Hom}\left(R / I, H_{I}^{0}(M, L)\right) \cong \operatorname{Hom}\left(M, \operatorname{Hom}\left(R / I, H_{I}^{0}(L)\right)\right)$, which is of finite length. By [13, Proposition 4.3], the result holds.

Suppose that $s>1$, and the result holds for the case $s-1$. The short exact sequence

$$
0 \longrightarrow H_{I}^{0}(L) \longrightarrow L \longrightarrow L / H_{I}^{0}(L) \longrightarrow 0
$$

yields the long exact sequence

$$
\cdots \longrightarrow H_{I}^{i}\left(M, H_{I}^{0}(L)\right) \longrightarrow H_{I}^{i}(M, L) \longrightarrow H_{I}^{i}\left(M, L / H_{I}^{0}(L)\right) \longrightarrow \cdots
$$

Since $H_{I}^{0}(L)$ is $I$-torsion, $H_{I}^{i}\left(M, H_{I}^{0}(L)\right) \cong \operatorname{Ext}_{R}^{i}\left(M, H_{I}^{0}(L)\right)$. Then, by Lemma 2.2, $H_{I}^{i}\left(M, H_{I}^{0}(L)\right)$ is $I$-cofinite and Artinian for all $i$. By [13, Corollary 4.4], it is enough for us to prove that $H_{I}^{i}\left(M, L / H_{I}^{0}(L)\right)$ is $I$-cofinite for all $i<s$. So we can assume that $\Gamma_{I}(L)=0$. Taking an injective hull $E$ of $L$, then we have the short exact sequence $0 \longrightarrow L \longrightarrow E \longrightarrow E / L \longrightarrow 0$. Consequently, from the long exact sequences of the above short exact sequence, $H_{I}^{i+1}(M, L) \cong H_{I}^{i}(M, E / L)$ and $H_{I}^{i+1}(L) \cong H_{I}^{i}(E / L)$ for all $i$. Thus, $H_{I}^{i}(E / L)$ is Artinian and $I$-cofinite, and $H_{I}^{i}(M, E / L)$ is Artinian for all $i<s-1$. Now by the induction hypothesis, the result is proved.

The following lemma has already been proved. However, we cannot find the original proof, so we give our own.

LEMMA 2.4. Assume that $0 \longrightarrow L_{1} \longrightarrow L_{2} \longrightarrow L_{3} \longrightarrow 0$ is an exact sequence of finitely generated $R$-modules. Then we have the long exact sequence

$$
\cdots \longrightarrow H_{I}^{i}\left(L_{3}, N\right) \longrightarrow H_{I}^{i}\left(L_{2}, N\right) \longrightarrow H_{I}^{i}\left(L_{1}, N\right) \longrightarrow H_{I}^{i+1}\left(L_{3}, N\right) \longrightarrow \cdots
$$


ProOf. Let $0 \longrightarrow N \longrightarrow E^{\bullet}$ be a minimal injective resolution of $N$. Note that $\Gamma_{I}(E)$ is injective if $E$ is injective. Then $\operatorname{Hom}\left(-, \Gamma_{I}(E)\right)$ is an exact functor. So $0 \longrightarrow \operatorname{Hom}\left(L_{3}, \Gamma_{I}\left(E^{\bullet}\right)\right) \longrightarrow \operatorname{Hom}\left(L_{2}, \Gamma_{I}\left(E^{\bullet}\right)\right) \longrightarrow \operatorname{Hom}\left(L_{1}, \Gamma_{I}\left(E^{\bullet}\right)\right) \longrightarrow 0$ is an exact sequence of $R$-complexes. By a well-known theorem of homology theory, we have a long exact sequence

$$
\begin{gathered}
\cdots \longrightarrow H^{i}\left(\operatorname{Hom}\left(L_{3}, \Gamma_{I}\left(E^{\bullet}\right)\right)\right) \longrightarrow H^{i}\left(\operatorname{Hom}\left(L_{2}, \Gamma_{I}\left(E^{\bullet}\right)\right)\right) \longrightarrow \\
H^{i}\left(\operatorname{Hom}\left(L_{1}, \Gamma_{I}\left(E^{\bullet}\right)\right)\right) \longrightarrow H^{i+1}\left(\operatorname{Hom}\left(L_{3}, \Gamma_{I}\left(E^{\bullet}\right)\right)\right) \longrightarrow \cdots .
\end{gathered}
$$

Suppose that $M$ is a finitely generated $R$-module. Then

$$
\Gamma_{I}\left(\operatorname{Hom}\left(M, E^{\bullet}\right)\right) \cong \operatorname{Hom}\left(M, \Gamma_{I}\left(E^{\bullet}\right)\right),
$$

and so

$$
\begin{aligned}
H_{I}^{i}(M, N) & =\underset{n}{\lim } \operatorname{Ext}_{R}^{i}\left(M / I^{n} M, N\right) \cong H^{i}\left(\Gamma_{I}\left(\operatorname{Hom}\left(M, E^{\bullet}\right)\right)\right) \\
& \cong H^{i}\left(\operatorname{Hom}\left(M, \Gamma_{I}\left(E^{\bullet}\right)\right)\right)
\end{aligned}
$$

Hence, we obtain the long exact sequence that we wished to prove.

For any submodule $K$ of a finitely generated $R$-module $L$, we use $K:_{L}\langle\mathfrak{m}\rangle$ to denote the submodule $\left\{x \in L \mid \mathfrak{m}^{n} x \subseteq K\right.$ for some $\left.n>0\right\}$. A sequence $x_{1}, \ldots, x_{n}$ of elements in $\mathfrak{m}$ is said to be an $\mathfrak{m}$-filter regular sequence on a module $N$ if

$$
\left(x_{1}, \ldots, x_{i-1}\right) N:_{N} x_{i} \subseteq\left(x_{1}, \ldots, x_{i-1}\right) N:_{N}\langle\mathfrak{m}\rangle
$$

for all $i=1, \ldots, n$. The f-depth of an ideal $I$ on a module $N$ is defined as the length of any maximal $\mathfrak{m}$-filter regular sequence on $N$ in $I$; we denote it by f-depth $(I, N)$. As the analogue of a result in the local cohomology modules case, the authors of [5] proved that $\mathrm{f}-\operatorname{depth}(I+\operatorname{Ann} M, N)$ is equal to $\operatorname{Min}\left\{r \mid H_{I}^{r}(M, N)\right.$ is not Artinian $\}$. On the other hand, from the definition of generalized local cohomology modules, it is well known that, for all $i$,

$$
H_{I+\operatorname{Ann} M+\operatorname{Ann} N}^{i}(M, N) \cong H_{I+\operatorname{Ann} M}^{i}(M, N) \cong H_{I}^{i}(M, N) .
$$

Now we are in the position to present our first main result.

THEOREM 2.5. Let $M$ and $N$ be two finitely generated $R$-modules. If, for some nonnegative integer $t, H_{I}^{i}(M, N)$ is Artinian for all $i<t$, then $H_{I}^{i}(M, N)$ is I-cofinite for all $i<t$ and $\operatorname{Hom}\left(R / I, H_{I}^{t}(M, N)\right)$ is finitely generated.

Proof. Since $H_{I}^{i}(M, N)$ is Artinian for all $i<t, H_{I+\operatorname{Ann} M}^{i}(N)$ is Artinian for all $i<t$ by [5, Theorem 2.2]. Then by Lemma 2.1, $H_{I+\operatorname{Ann} M}^{i}(N)$ is $(I+\operatorname{Ann} M)$ cofinite for all $i<t$. Therefore, for any $i<t, H_{I+\operatorname{Ann} M}^{i}(M, N)$ is $(I+\operatorname{Ann} M)$ cofinite by Lemma 2.3. In particular, $\operatorname{Hom}\left(R /(I+\operatorname{Ann} M), H_{I+\operatorname{Ann} M}^{i}(M, N)\right)$ is 
finitely generated for all $i<t$. Note that $\operatorname{Hom}\left(R / I, H_{I}^{i}(M, N)\right) \cong \operatorname{Hom}(R /(I+$ Ann $\left.M), H_{I+\operatorname{Ann} M}^{i}(M, N)\right)$, so that $\operatorname{Hom}\left(R / I, H_{I}^{i}(M, N)\right)$ is finitely generated for all $i<t$. Thus, by [13, Proposition 4.3], it follows that $H_{I}^{i}(M, N)$ is $I$-cofinite for all $i<t$ from the hypothesis that $H_{I}^{i}(M, N)$ is Artinian for all $i<t$.

In the following, we prove that $\operatorname{Hom}\left(R / I, H_{I}^{t}(M, N)\right)$ is finitely generated. Since, for any $i$,

$$
H_{I+\operatorname{Ann} M}^{i}(M, N) \cong H_{I}^{i}(M, N)
$$

and

$$
\operatorname{Hom}\left(R / I, H_{I}^{i}(M, N)\right) \cong \operatorname{Hom}\left(R /(I+\operatorname{Ann} M), H_{I+\operatorname{Ann} M}^{i}(M, N)\right),
$$

we can assume that Ann $M \subseteq I$.

Let $0 \longrightarrow K \longrightarrow R^{n} \longrightarrow M \longrightarrow 0$ be an exact sequence of finitely generated $R$ modules. By Lemma 2.4, we have the exact sequence

$$
\cdots \longrightarrow H_{I}^{t-1}(K, N) \stackrel{\alpha}{\longrightarrow} H_{I}^{t}(M, N) \longrightarrow H_{I}^{t}\left(R^{n}, N\right) \longrightarrow \cdots .
$$

It is clear that $H_{I}^{i}\left(R^{n}, N\right) \cong \bigoplus_{i=1}^{n} H_{I}^{i}(N)$ for any $i$. Then $H_{I}^{i}\left(R^{n}, N\right)$ and $H_{I}^{i}(K, N)$ are Artinian for all $i<t$ by [5, Theorem 2.2]. By virtue of the former part of the result, we know that $H_{I}^{i}(K, N)$ is $I$-cofinite for all $i<t$. Let $L$ denote the image of $\alpha$ in the above long exact sequence. By [13, Corollary 4.4], $L$ is $I$-cofinite. From the exact sequence

$$
0 \longrightarrow L \stackrel{\alpha}{\longrightarrow} H_{I}^{t}(M, N) \longrightarrow H_{I}^{t}\left(R^{n}, N\right) \longrightarrow \cdots,
$$

we have the exact sequence

$$
0 \longrightarrow \operatorname{Hom}(R / I, L) \longrightarrow \operatorname{Hom}\left(R / I, H_{I}^{t}(M, N)\right) \longrightarrow \operatorname{Hom}\left(R / I, H_{I}^{t}\left(R^{n}, N\right)\right) .
$$

By Lemma 2.1, the right term of the above exact sequence is finitely generated. Then the result follows from the above exact sequence.

The following lemma is a generalization of [14, Lemma 3.4].

Lemma 2.6 ([12, Theorem 3.2]). Let $M$ be a finitely generated $R$-module such that $d=\operatorname{pd}(M)<\infty$. Let $N$ be a finitely generated $R$-module and assume that $n$ is an integer, and $x_{1}, x_{2}, \ldots, x_{n}$ is an I-filter regular sequence on $N$. Then

$$
H_{I}^{i+n}(M, N) \cong H_{I}^{i}\left(M, H_{\left(x_{1}, x_{2}, \ldots, x_{n}\right)}^{n}(N)\right)
$$

for all $i \geq d$.

Proposition 2.7. Let I be an ideal of $R$, and let $M, N$ be two finitely generated $R$-modules such that $d=\operatorname{pd}(M)<\infty$ and $\operatorname{dim} N=n<\infty$. Then $H_{I}^{d+n}(M, N) \cong$ $\operatorname{Ext}_{R}^{d}\left(M, H_{I}^{n}(N)\right)$. In particular, $H_{I}^{d+n}(M, N)$ is Artinian. 
PROOF. For this integer $n$, it is well known that there exists a sequence $x_{1}, x_{2}, \ldots, x_{n}$ in $I$ such that it is an $I$-filter regular sequence on $N$. Note that $H_{\left(x_{1}, x_{2}, \ldots, x_{n}\right)}^{n}(N)$ is Artinian when $n=\operatorname{dim} N$. By virtue of [14, Lemma 3.4],

$$
H_{\left(x_{1}, x_{2}, \ldots, x_{n}\right)}^{n}(N) \cong H_{I}^{0}\left(H_{\left(x_{1}, x_{2}, \ldots, x_{n}\right)}^{n}(N)\right) \cong H_{I}^{n}(N) .
$$

Therefore, by Lemma 2.6,

$$
H_{I}^{d+n}(M, N) \cong H_{I}^{d}\left(M, H_{\left(x_{1}, x_{2}, \ldots, x_{n}\right)}^{n}(N)\right) \cong H_{I}^{d}\left(M, H_{I}^{n}(N)\right) \cong \operatorname{Ext}_{R}^{d}\left(M, H_{I}^{n}(N)\right) .
$$

This completes the proof.

The following theorem is our second main result, which generalizes [6, Theorem 3].

THEOREM 2.8. Let I an ideal of $R$, and let $M, N$ be two finitely generated $R$-modules such that $d=\operatorname{pd}(M)<\infty$ and $\operatorname{dim} N=n<\infty$. Then $H_{I}^{d+n}(M, N)$ is I-cofinite.

Proof. By [6, Theorem 3], we know that $H_{I}^{n}(N)$ is $I$-cofinite. Then by Lemma 2.2 and Proposition 2.7, the result follows.

In the last part of this note, we discuss the finiteness of $H_{I}^{i}(M, N)$.

LEMMA 2.9. Let $N$ be a finitely generated $R$-module and $M$ a nonzero cyclic $R$-module. Let $t$ be a positive integer. If $H_{I}^{i}(N)$ is finitely generated for all $i<t$, then $H_{I}^{t}(N)$ is finitely generated if and only if $\operatorname{Hom}\left(M, H_{I}^{t}(N)\right)$ is finitely generated.

PROOF. The 'only if' part is clear. Now suppose that $\operatorname{Hom}\left(M, H_{I}^{t}(N)\right)$ is finitely generated. Note that $\operatorname{Hom}\left(M, H_{I}^{t}(N)\right)$ is $I$-torsion, then there exists an integer $n$ such that $I^{n} \operatorname{Hom}\left(M, H_{I}^{t}(N)\right)=0$. Assume that $M$ is generated by an element $m$. For any $x \in H_{I}^{t}(N)$, we can find an element $f \in \operatorname{Hom}\left(M, H_{I}^{t}(N)\right)$ such that $f(m)=x$. Since $I^{n} f=0, I^{n} x=0$, and so $I^{n} H_{I}^{t}(N)=0$. Since $H_{I}^{i}(N)$ is finitely generated for all $i<t$, by [3, Proposition 9.1.2], there exists an integer $r, I^{r} H_{I}^{i}(N)=0$ for all $i<t$. Thus, $I^{r} H_{I}^{i}(N)=0$ for all $i<t+1$. Again by [3, Proposition 9.1.2], $H_{I}^{i}(N)$ is finitely generated for all $i<t+1$. In particular, $H_{I}^{t}(N)$ is finitely generated.

Proposition 2.10. Let $N$ be a finitely generated $R$-module and let $t$ be a positive integer. If $M$ is a nonzero cyclic $R$-module, then $H_{I}^{i}(N)$ is finitely generated for all $i<t$ if and only if $H_{I}^{i}(M, N)$ is finitely generated for all $i<t$.

PROOF. The 'only if' part has been proved in [11, Theorem 1.1(iv)]. Now we suppose that $H_{I}^{i}(M, N)$ is finitely generated for all $i<t$. By induction on $t$, we can assume that $H_{I}^{i}(N)$ is finitely generated for all $i<t-1$. Then by [11, Theorem 1.1(iii)], it follows that $\operatorname{Hom}\left(M, H_{I}^{t-1}(N)\right)$ is finitely generated from the fact that $H_{I}^{t-1}(M, N)$ is finitely generated. Then $H_{I}^{t-1}(N)$ is finitely generated by Lemma 2.9. 


\section{Acknowledgement}

The author would like to thank Professor Z. M. Tang for many stimulating discussions and much encouragement in writing this paper.

\section{References}

[1] J. Asadollahi, K. Khashyarmanesh and Sh. Salarian, 'On the finiteness properties of the generalized local cohomology modules', Comm. Algebra 30 (2002), 859-867.

[2] M. H. Bijan-Zadeh, 'A common generalization of local cohomology theories', Glasgow Math. J. 21 (1980), 173-181.

[3] M. P. Brodmann and R. Y. Sharp, Local Cohomology: An Algebraic Introduction with Geometric Applications (Cambridge University Press, Cambridge, 1998).

[4] W. Bruns and J. Herzog, Cohen-Macaulay Rings, Revised edn (Cambridge University Press, Cambridge, 1996).

[5] L. Z. Chu and Z. M. Tang, 'On the Artinianness of generalized local cohomology', Comm. Algebra 35 (2007), 3821-3827.

[6] D. Delfino and T. Marley, 'Cofinite modules and local cohomology', J. Pure Appl. Algebra 121 (1997), 45-52.

[7] M. T. Dibaei and S. Yassemi, 'Associated primes and cofiniteness of local cohomology modules', Manuscripta Math. 117 (2005), 199-205.

[8] K. Divaani-Aazar and R. Sazeedeh, 'Cofiniteness of generalized local cohomology modules', Colloq. Math. 99 (2004), 283-290.

[9] R. Hartshorne, 'Affine duality and cofiniteness', Invent. Math 9 (1970), 145-164.

[10] J. Herzog, Komplexe Auflösungen und Dualität in der lokalen Algebra, Universität Regensburg, Preprint, 1974.

[11] K. Khashyarmanesh and M. Yassi, 'On the finiteness property of generalized local cohomology modules', Algebra Colloq. 12 (2005), 293-300.

[12] K. Khashyarmanesh, M. Yassi and A. Abbasi, 'Filter regular sequence and generalized local cohomology modules', Comm. Algebra 32 (2004), 253-259.

[13] L. Melkersson, 'Modules cofinite with respect to an ideal', J. Algebra 285 (2005), 649-668.

[14] U. Nagel and P. Schenzel, 'Cohomological annihilators and Castelnuovo Mumford regularity', in: Commutative Algebra: Syzygies, Multiplicities, and Birational Algebra (South Hadley, MA, 1992) (American Mathematical Society, Providence, RI, 1994), pp. 307-328.

[15] J. Rotman, Introduction to Homological Algebra (Academic Press, New York, 1979).

[16] N. Suzuki, 'On the generalized local cohomology and its duality', J. Math. Kyoto. Univ. 18 (1978), 71-85.

[17] S. Yassemi, 'Cofinite modules', Comm. Algebra 29 (2001), 2333-2340.

LIZHONG CHU, Department of Mathematics, Suzhou University, 215006, Jiangsu, PR China

e-mail: Chulizhong@suda.edu.cn 